\title{
La forme territoriale de la Suisse: Essai quantitatif en géographie politique
}

La surface terrestre est un palimpseste d'unités spatiales allant des océans et des continents aux champs et - aux bâtisses. Toutes ont des limites qui peuvent être visibles (clôture d'un jardin) ou invisibles (limite municipale). L'Etat est l'une de ces unités spatiales puisqu'il est constitué d'un territoire ceinture de frontières. Une qualité des territoires étatiques, qui s'impose à la lecture de toute carte politique, est leur forme (contour spatial ou allure). Or, la forme a toujours été un centre d'intérêt en géographiel). En effet, beaucoup d'éléments géographiques comme les deltas, les cônes de déjection, les chapelets insulaires sont ainsi nommés à cause de leurs formes distinctives. La forme est aussi utile pour les territoires politiques car elle est une des propriétés les plus significatives des surfaces spatiales. La Suisse n'a pas atteint ses propriétés territoriales d'un seul coup. Sa forme actuelle est le résultat d'une longue séquence historique d'ajustements et de réajustements (pertes ou gains de territoire). Quand, de prime abord, l'on considère la carte de plusieurs pays, il y a tant de genres différents de forme territoriale qu'une classification semble impossible. Toutefois, d'un point de vue uniquement qualitatif, la géographie politique classique distingue six catégories de formes territoriales: Etat allongé, Etat fragmenté, Etat perforé, Etat étranglé, Etat compact, Etat appendiculaire2). Tous ces qualificatifs n'ont d'intérêt que par les conséquences qu'ils engendrent au titre de la circulation, du contrôle gouvernemental, de la cohésion sociétale, de l'homogénéité culturelle, de la capitale politique, de l'irrédentisme ou du séparatisme... A l'aide de cette classification qualitative aux limites assez vagues, on peut définir la Suisse comme un Etat compact-appendiculaire dans la mesure où la compacité centrale de ce pays est contrebalancée par des appendices de détail, des sortes de tentacules qui se projettent loin en avant du corps principal du pays (Schaffhouse, Ajoie, Genève, Tessin, vallées periphériques des Grisons). Les classes qualitatives de formes d'Etats n'étant pas, en soi, des limites strictes, la Suisse représente incontestablement un type mixte.

Toutefois, comme le montre fort bien le résultat qualitatif, la forme territoriale est peut-être l'élément du "pattern" géographique le plus difficile à mesurer. En effet, comme on vient de le voir, elle est subjectivement décrite par comparaison avec certains objets existants et ceci donne des termes comme appendiculaire, étoilé, digité, lobé... Quelquefois sont employés des adjectifs peu précis comme allongé, étranglé, perforé, compact... Aucun de ces termes, cependant, ne donne une base précise pour la comparaison.

L'approche quantitative est donc une nécessité puisqu'elle permet une définition plus statistique et plus rigoureuse de la forme territoriale de la Confédération Helvétique. De 1941 à 1970, quatre méthodes ont été développées par les géographes pour mesurer la forme. A travers ces méthodes, se dégageront des indices de forme pour la Suisse qu'il conviendra de comparer avec d'autres Etats très petits de l'Europe pour qu'ils prennent pleine signification.

Le premier et le plus ancien procédé est la méthode KRUMBEIN-PETTIJOHN (1941-1957). Il s'agit d'une méthode à 5 indices autonomes qui, en principe, doivent arriver au même résultat, c'est-à-dire définir la forme3).

Le premier indice est:

$$
\mathrm{S}_{1} \frac{\mathrm{A}}{0,282 \mathrm{P}}
$$

où $\mathrm{A}$ est la superficie de l'Etat concerné en $\mathrm{km}^{2}$ et $\mathrm{P}$, le périmètre du même Etat (en $\mathrm{km}$ ).

Le deuxième indice est

$$
\mathrm{S}_{2}=\frac{\mathrm{A}}{0,866 \mathrm{~L}}
$$

où $\mathrm{L}$ est le plus long axe intérieur.

Le troisième indice est

$$
\mathrm{S}_{3}=\frac{\mathrm{R}_{1}}{\mathrm{R}_{2}}
$$

où $\mathrm{R}_{1}$ est le rayon du plus grand cercle inscrit à l'intérieur du territoire de l'Etat et $\mathrm{R}_{2}$ le rayon du plus petit cercle extérieur.

Le quatrième indice est

$$
\mathrm{S}_{4}=\frac{\mathrm{A}}{\pi(0,5 \mathrm{~L})^{2}}
$$

Prof. André-Louis Sanguin, Directeur, Département de Géographie et Pierre Gauthier, Professeur de géographie quantitative, Université du Québec à Chicoutimi, 930 est, rue Jacques Cartier, Chicoutimi, Québec G 7H 2B1, Canada 
Fig. 1 Méthode Krumbein-Pettijohn

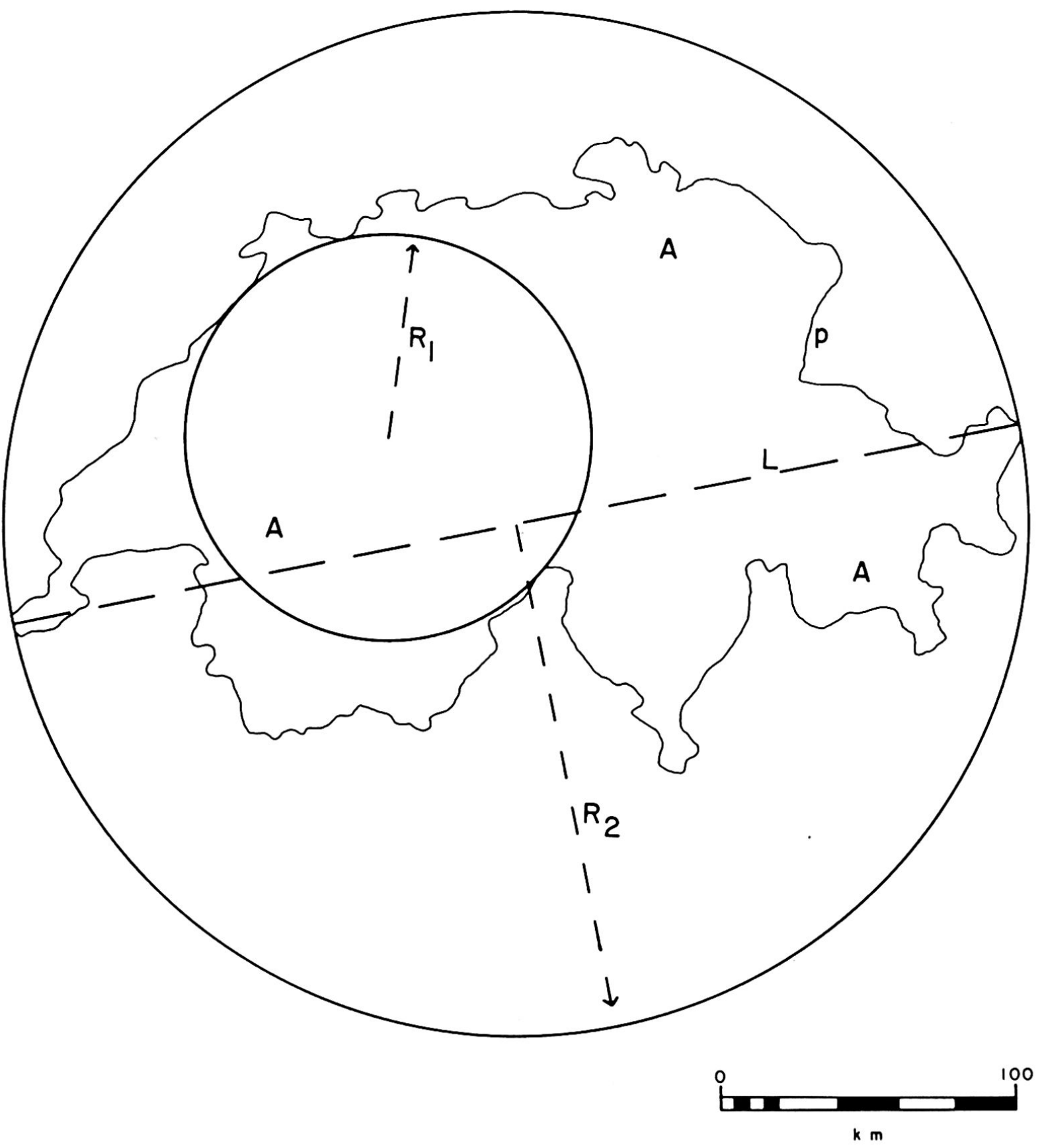


et le cinquième indice est

$$
\mathrm{S}_{5}=\frac{1,27 \mathrm{~A}}{\mathrm{~L}}
$$

Il est inutile d'utiliser l'indice $S_{5}$ en supplément de l'indice $S_{2}$ dans la mesure où $S_{4}$ equivaut à 1,10 fois l'indice $\mathrm{S}_{2}$.
De façon à rendre plus explicite l'application de la méthode KRUMBEIN-PETTIJOHN à la forme de la Suisse (cf. figure 1), il est nécessaire de la comparer avec celle d'autres Etats de même taille (Belgique, Hongrie, Portugal, Autriche). De la même manière, la comparaison a été appliquée à des figures géométriques de superficie semblable aux cinq pays (carré, rectangle, triangle). Les résultats sont donc les suivants:

\begin{tabular}{|l|c|c|c|c|}
\hline Pays & $\mathrm{S}_{1}$ & $\mathrm{~S}_{2}$ & $\mathrm{~S}_{3}{ }^{*}$ & $\mathrm{~S}_{4}$ \\
\hline Suisse & 111,129 & 141,264 & 0,531 & 0,461 \\
\hline Belgique & 104,603 & 122,654 & 0,45 & 0,47 \\
\hline Hongrie & 207,51 & 210,637 & 0,451 & 0,455 \\
\hline Portugal & 168,97 & 181,76 & 0,271 & 0,342 \\
\hline Autriche & 159,858 & 172,899 & 0,389 & 0,340 \\
\hline Formes géométriques & $\mathrm{S}_{1}$ & $\mathrm{~S}_{2}$ & $\mathrm{~S}_{3}{ }^{*}$ & $\mathrm{~S}_{4}$ \\
\hline Carré & 181,73 & 168,792 & 0,702 & 0,647 \\
\hline Grand carré** & 363,475 & 349,749 & 0,713 & 0,695 \\
\hline Rectangle & 175,873 & 158,242 & 0,457 & 0,506 \\
\hline Grand rectangle** & 363,475 & 330,871 & 0,449 & 0,553 \\
\hline Triangle & 121,158 & 118,36 & 0,5 & 0,637 \\
\hline Grand triangle** & 242,317 & 236,72 & 0,511 & 0,637 \\
\hline
\end{tabular}

* Dans le cas de $S_{3}$, le chiffre 1 est égal au cercle parfait.

** Le grand carré, le grand rectangle et le grand triangle représentent approximativement deux fois la superficie respectivement du carré, du rectangle et du triangle.

En ce qui concerne $S_{3}$, il est intéressant de mettre en relation l'indice résultant pour la Suisse et les indices des formes géométriques abstraites. L'indice des formes géométriques varie sensiblement dès qu'il $\mathrm{y} a$ une modification de l'ordre de grandeur des formes. Il en va de même pour les cinq Etats pris globalement: une même forme mais avec superficie doublée aboutit à des indices différents. Toutefois, avec $\mathrm{S}_{3}$, le territoire suisse montre des échappées triangulaires vers l'extérieur sans pour autant qu'on puisse aboutir à un indice précis définissant la forme exacte de la Confédération.

En ce qui concerne $\mathrm{S}_{4}$, LEE et SALLEE posent le postulat que chaque forme doit avoir son indice propre4). Or, 
dans l'alternative $\mathrm{S}_{4}$, les indices sont trop proches, voire même semblables; avec une telle formule, la Suisse, la Belgique et la Hongrie ont des tendances circulaires et des formes similaires. Par contre, le Portugal et l'Autriche, plutôt de forme rectangulaire, ressortent avec une non-correspondance entre les résultats et les indices de formes géométriques. Donc, dans l'alternative $\mathrm{S}_{4}$, il est nécessaire de travailler sur d'autres formes géométriques pour trouver des indices correspondant aux pays.

En ce qui concerne $S_{1}, S_{2}$ et $S_{5}$, il apparaît clairement que ce ne sont pas des indices réellement utilisables dans la mesure où une même forme aboutit à des écarts d'indice (par exemple, le rectangle). Un tel type d'indice tient trop compte de l'ordre de grandeur (dans ces formules, on utilise essentiellement la superficie). Il est évident que dans les cas de $S_{1}, S_{2}$ et $S_{5}$, il n'y a aucun lien entre la théorie et la réalité.

Le deuxième procédé est la méthode BUNGE (1962). C'est une méthode très longue où l'indice final est la somme des indices sommaires5). Elle est basée sur l'utilisation d'un polygone de côtés égaux qui représente, au mieux, la forme à étudier comme le montre la figure 26).

Le premier indice sommaire se calcule en additionnant les vecteurs $1-3,2-4,3-5$ et ainsi de suite... Le deuxième indice sommaire s'obtient par l'addition des vecteurs 1-4, 2-5, 3-6 et ainsi de suite pour tous les autres indices sommaires. L'indice sommaire au carré est la somme de chacun des vecteurs au carré. Pour un ensemble aussi disparate que la Suisse et les quatre autres Etats comparés, il est extrêmement difficile d'arriver à un même nombre de vecteur par pays. Le tableau ci-dessous donne les résultats suivants:
Fig. 2 Méthode Bunge
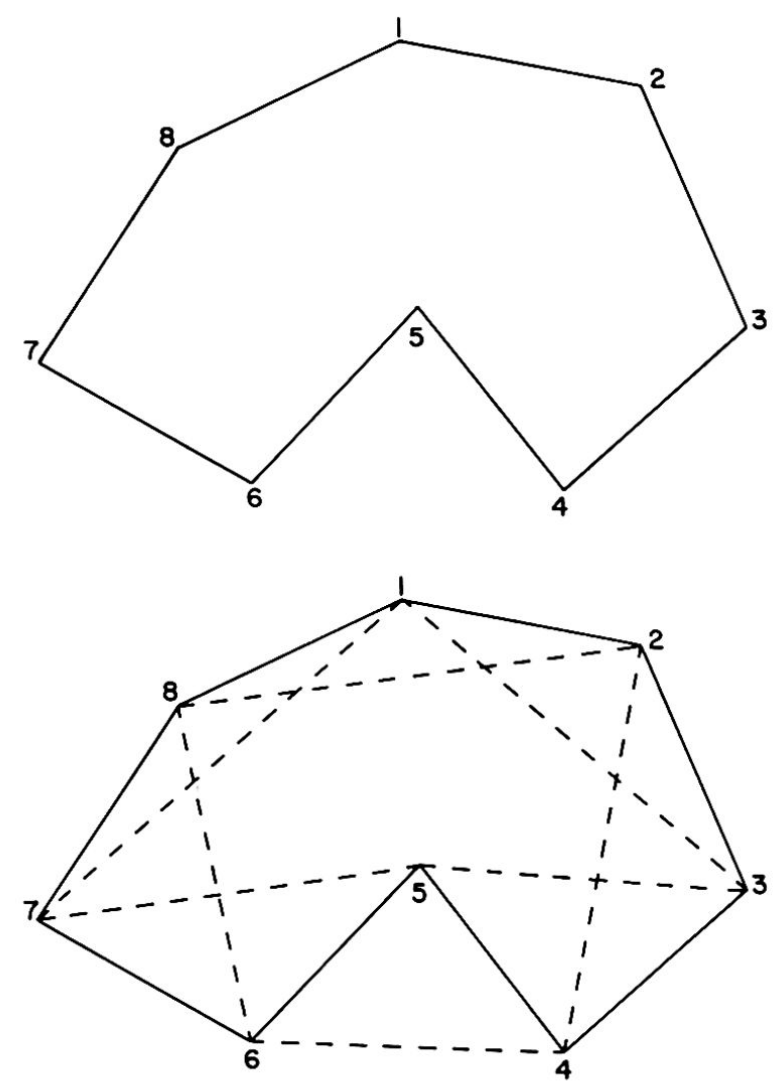

\begin{tabular}{l|llllll|lllllll|l}
\hline Pays & \multicolumn{3}{|l|}{ Indice sommaire } & & & & \multicolumn{9}{|l|}{ Indice sommaire au carré } & & \multicolumn{2}{l}{$\begin{array}{l}\text { Vec- } \\
\text { teurs }\end{array}$} \\
\hline & 1 & 2 & 3 & 4 & 5 & 6 & 12 & 22 & 33 & 42 & 52 & 62 & \\
Suisse & 19,75 & 27,65 & 33,13 & 36 & & & 37,00 & 71,18 & 102,72 & 122,16 & & & 11 \\
Autriche & 17,28 & 22,86 & 29,86 & 26,76 & & & 31,91 & 56,44 & 75,76 & 82,22 & & & 10 \\
Belgique & 26,80 & 38,00 & 47,20 & 53,95 & 59,2 & 61,7 & 48,52 & 98,56 & 153,98 & 202,67 & 244,92 & 267,79 & 15 \\
Portugal & 23,48 & 31,73 & 38,74 & 43,42 & 45,80 & & 43,34 & 80,85 & 123,62 & 160,39 & 181,32 & & 13 \\
Hongrie & 24,50 & 34,10 & 41,95 & 46,73 & 49,71 & & 46,88 & 91,61 & 140,46 & 176,73 & 201,06 & & 13 \\
\hline
\end{tabular}


La difficulté majeure à laquelle se heurte la méthode BUNGE est le nombre des indices sommaires. Plus on veut de précision pour définir la forme, plus il faut d'indices sommaires. Par ailleurs, plus il y a de vecteurs, plus les indices sommaires sont élevés. En réalité, la méthode BUNGE n'est pas révélatrice de formes territoriales parce que les résultats chiffrés ne sont aucunement rattachés à un qualificatif quelconque. En comparant 150 formes ensemble, on pourrait peut-être arriver à définir certains indices mais la comparaison est extrêmement difficile lorsqu'il s'agit d'un petit nombre de territoires d'Etats.

Le troisième procédé est la méthode BOYCE-CLARK (1964). Il s'agit d'une méthode où il faut utiliser de façon exclusive le centre géométrique du territoire impliqué pour définir la forme7). La formule d'obtention de l'indice est:

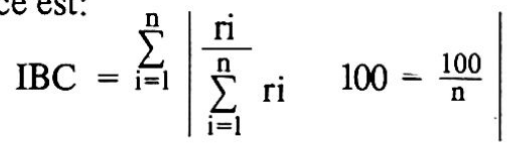

où IBC est l'indice BOYCE-CLARK, $r i$ la longueur du rayon $i, n$ le nombre de rayons et \| l'expression mathématique signifiant «valeur absolue de».

Mentionnons que le centre géométrique de la Suisse, d'après le Service Topographique Fédéral, est situé dans le Klein Melchtal à $10 \mathrm{~km}$ au Sud de Sarnen (Obwalden). A partir du centre géométrique, on construit une série de rayons équidistants allant jusqu'au périmètre du territoire politique (Figure 3). L'utilisa-

Fig. 3 Méthode Boyce-Clark

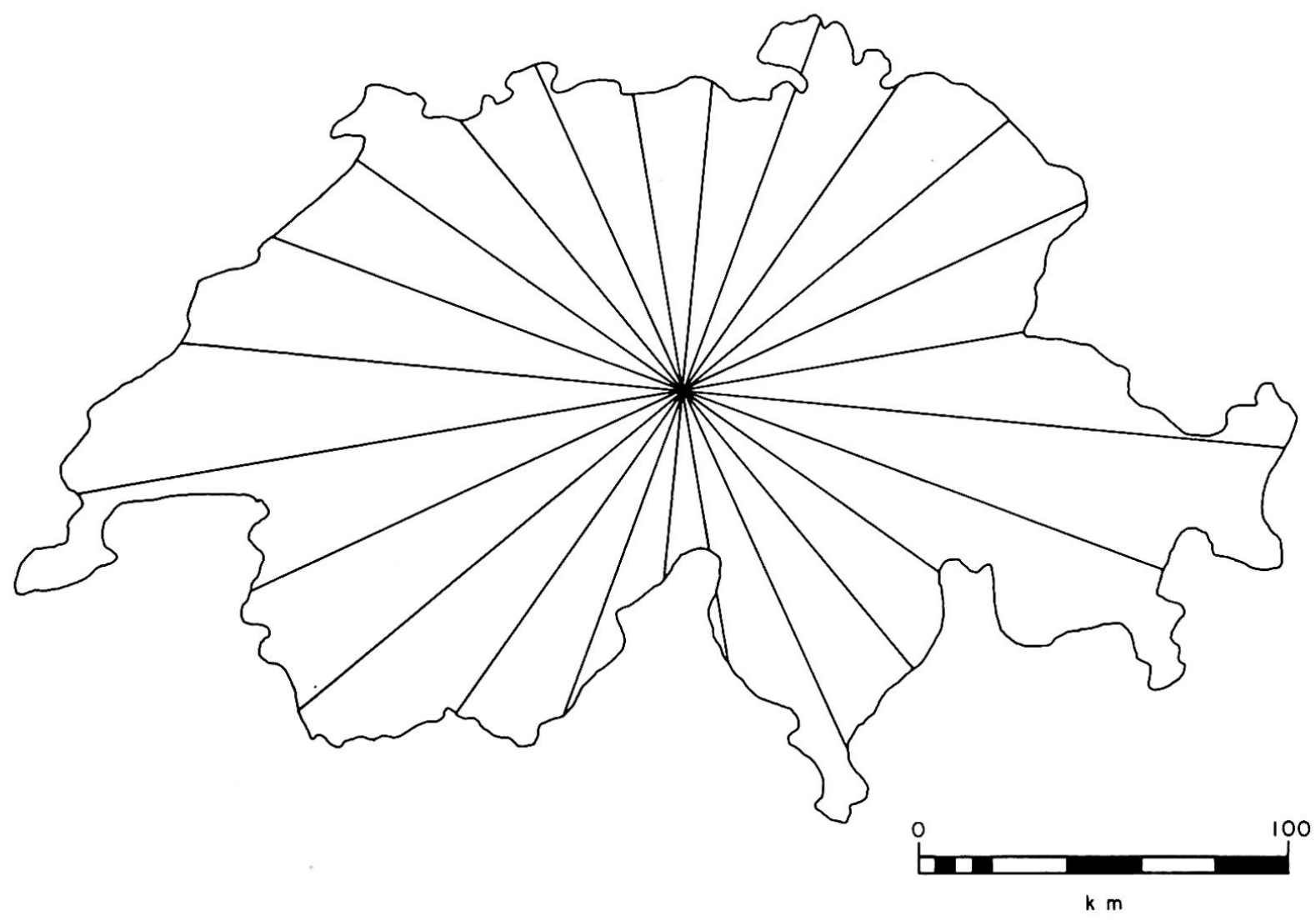


tion d'un grand nombre de rayons apporte une plus grande exactitude. Chaque rayon équivaut à $\mathrm{X} \%$ de la somme de tous les rayons. La somme des différences entre le pourcentage de chacun des rayons et la valeur moyenne des rayons est l'indice BOYCE-CLARK.
Au départ, BOYCE et CLARK donnent les indices correspondant aux principales formes géométriques (cercle, carré, croix, triangle, étoile, rectangle, ligne...). Pour la Suisse et les autres Etats comparés, les indices résultés apparaissent dans le tableau suivant:

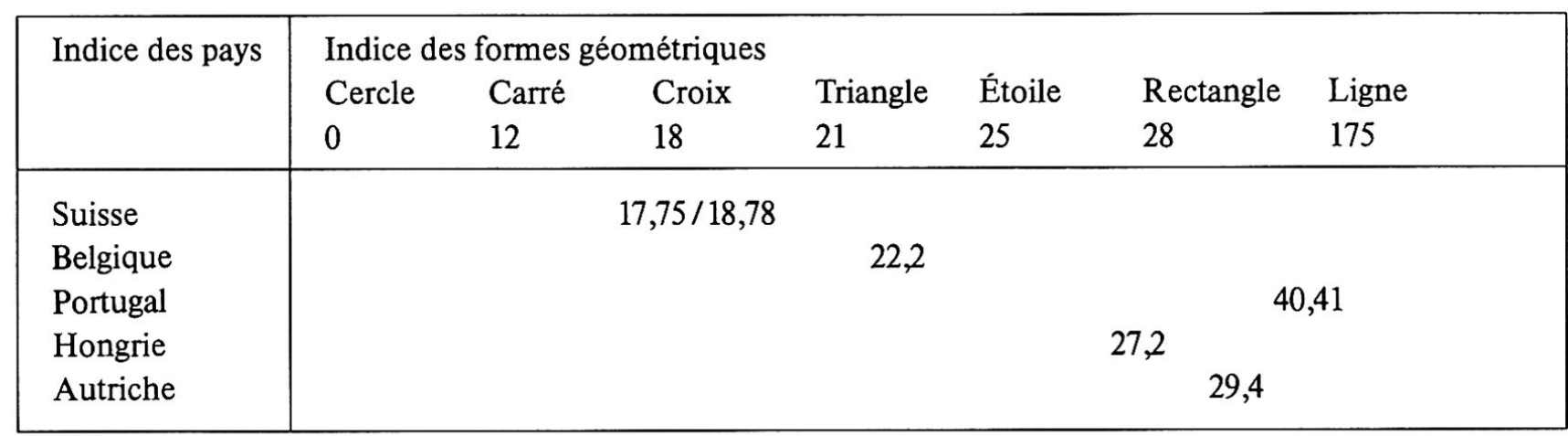

Cette méthode revêt certains avantages. L'indice de base est assez précis pour chacune des formes, peu importe l'ordre de grandeur; en d'autres termes, le calcul n'est pas troublé par la superficie. Dans la méthode BOYCE-CLARK, l'impression visuelle est corroborée par les indices avec des qualificatifs appropriés. Toutefois, LEE et SALLEE estiment qu'on peut arriver à des indices identiques pour des formes différentes à cause du trop petit nombre de vecteurs employés8). Par contre, si l'on reprend les mêmes formes mais avec le double de vecteurs, on arrive réellement à définir les différences.
Le quatrième et dernier procédé est la méthode LEESALLEE (1970)9). La formule d'obtention d'indice est:

$\mathrm{ILS}=1-\frac{\text { surface }(\mathrm{K} \cap L)}{\text { surface }(\mathrm{KUL})}$

où ILS est l'indice LEE-SALLEE, $\mathrm{K}$ représente la forme du territoire, $L$ la forme géométrique abstraite à appliquer, $\Omega$ l'expression mathématique signifiant «intersection de» et $U$ l'expression mathématique signifiant «réunion de». A partir du moment où l'on obtient les indices se rapportant à chacune des formes géométriques, l'indice le plus bas devient celui qui

\section{Fig. 4 Méthode Lee-Salee}

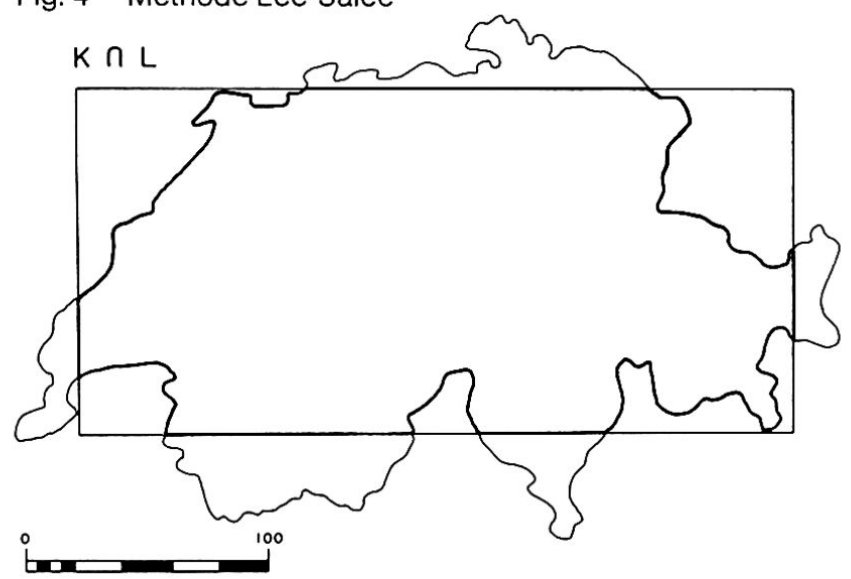
$\mathrm{xm}$
Fig. 5 Méthode Lee-Salee

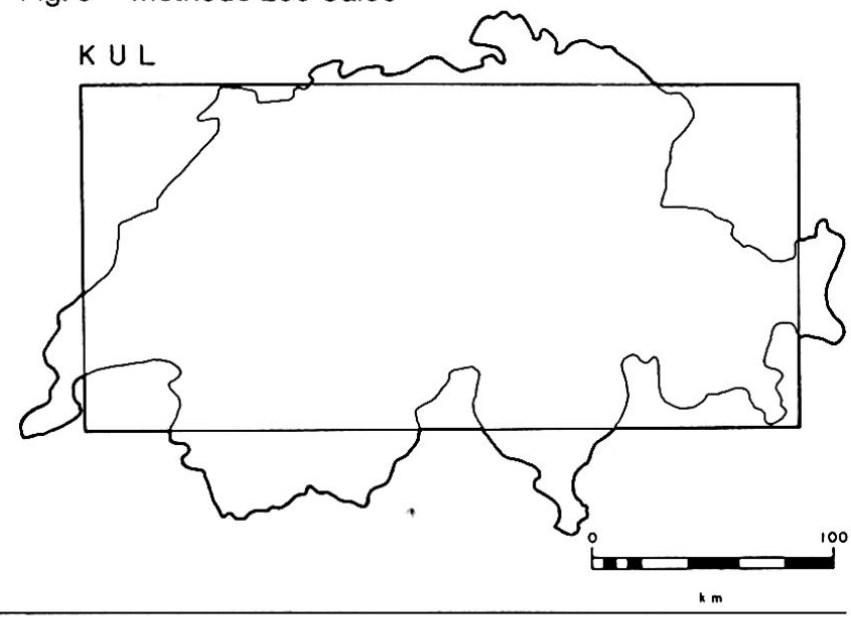


ajuste au mieux la forme du territoire. Si, par exemple, on met en rapport un rectangle avec la forme territoriale de la Suisse, on obtient $\mathrm{K} \cap \mathrm{L}$ avec la figure 4 et KUL avec la figure 5.
En égard à trois formes abstraites (cercle, rectangle, carré), les résultats pour la Suisse et les autres Etats comparatifs s'expriment dans le tableau suivant:

\begin{tabular}{|c|c|c|c|c|c|}
\hline Forme & $\begin{array}{l}\text { Pays } \\
\text { Belgique }\end{array}$ & Suisse & Portugal & Hongrie & Autriche \\
\hline Carré & 0,388 & 0,363 & 0,584 & 0,393 & 0,391 \\
\hline Rectangle & 0,357 & 0,316 & 0,298 & 0,233 & 0,369 \\
\hline Cercle & 0,309 & 0,323 & 0,551 & 0,394 & 0,385 \\
\hline
\end{tabular}

L'un des impératifs de la méthode est qu'il faut que la forme géométrique abstraite soit de même superficie que le territoire politique dont on cherche la forme. La principale critique relevée contre cette méthode réside dans le fait que les indices sont trop proches les uns des autres pour que l'on puisse concéder l'avantage à une forme plutôt qu'à une autre. C'est une méthode longue et son manque d'exactitude l'empêche d'être opératoire. Par ailleurs, les résultats numériques n'ont pas de rapport avec l'impression visuelle.

\section{Conclusion}

En résumé, deux questions fondamentales se posent:

a) quelle est la meilleure méthode pour définir la forme d'un territoire politique quelconque?

b) quelle est la tendance de la forme du territoire suisse, eu égard à la meilleure méthode et eu égard aux quatre autres Etats comparatifs?

La méthode KRUMBEIN-PETTIJOHN à le tort de trop tenir compte de la superficie. Bien que, des cinq indices de cette méthode, $S_{3}$ semble celui qui est le plus utilisable, on peut toutefois douter de son exactitude et c'est donc une méthode qui n'apparaît pas révélatrice de formes spécifiques. Les méthodes BUNGE et LEE-SALLEE laissent à désirer dans la mesure où il $s$ 'agit d'appliquer des formes géométriques abstraites sur un territoire politique. On en arrive à des approximations bien trop vagues. Il reste la méthode BOYCECLARK. Son calcul a l'avantage de ne pas être troublé par la superficie, contrairement à la méthode KRUM-
BEIN-PETTIJOHN. Même s'il s'agit de l'application de rayons, ceux-ci ont comme point d'arrêt le périmètre du territoire politique et non celui de la forme géométrique abstraite, ce qui est beaucoup plus réaliste. Une preuve corrobore cette affirmation: si, en gardant le même centre géographique d'un Etat, on déplace les rayons, les indices obtenus demeurent pratiquement les mêmes (pour la Suisse: 18,78 dans un cas et 17,75 dans l'autre). Donc, mutatis mutandi, c'est la méthode qui demeure la plus appropriée et la plus utilisable pour des formes politico-territoriales qui, par nature, sont irrégulières. Toutefois, dans cette méthode, il faut toujours partir du centre géométrique du pays.

Dès lors, quelle est la forme territoriale de la Suisse? Selon le procédé BOYCE-CLARK, l'indice d'une allure carrée est 12 , celui d'une allure cruciforme 18 et celui d'une allure triangulaire 21 . Or, suivant cette méthode de calcul, la Suisse obtient l'indice 17,75 ou 18,78 selon que l'on change la direction des rayons à partir du centre géométrique. On peut donc en déduire que la Confédération possède un centre de forme carrée avec, sur son pourtour, des échappées triangulaires, ce qui aboutit par combinaison, à une allure cruciforme. L'approche quantitative permet donc de renforcer la classification qualitative définissant la Suisse comme un Etat de forme compacte-appendiculaire. Toutefois, l'analyse que nous venons de proposer démontre que les méthodes quantitatives appliquées à des aspects particuliers de la géographie politique ne font pas apparaître automatiquement des concordances sûres et précises entre le modèle théorique et la forme du territoire étudié (ainsi, selon la méthode KRUMBEIN-PETTIJOHN, le rectangle a un indice de 0,449 tandis 
que le Portugal obtient 0,271). Certains doutes subsistent donc quant aux résultats issus de telle ou telle formule lorsqu'on les compare avec la réalité visuelle: ainsi, l'on est en droit de se demander si vraiment, comme le voudrait la méthode LEE-SALLEE, l'Autriche est un rectangle et la Belgique un cercle. Ces difficultés de quantification peuvent être acceptées soit comme l'évidence d'une lacune dans la mesure des formes, soit comme l'incapacité de l'homme de distinguer, à l'œil nu, les différences de forme. Cependant, le point le plus important de cette étude n'était pas un commentaire sur la forme territoriale de la Suisse comme telle, mais l'illustration des possibilités de mesurer les allures spatiales du territoire helvétique. Jusqu'ici, celles-ci pouvaient seulement être cartographiées. Une telle tentative d'illustration cherche à libérer la géographie politique d'une dépendance de l'utilisation subjective des cartes 10 ).

\section{Références bibliographiques}

1) KING, LESLIE J., «The Analysis of Spatial Form and Its Relations to Geographic Theory», Annals of the Association of American Geographers, 1969, vol. 59, p. 573-595.
2) BLIJ, HARM J. DE, Systematic Political Geography, New York, John Wiley and Sons Inc., 1973 (2e édition), p. 38-43.

3) KRUMBEIN, WILliam C., «Measurement and Geologic Significance of Shape and Roundness of Sedimentary Particles», Journal of Sedimentary Petrology, 1941, vol. 11, p. 64-72.

PETTIJOHN, FRANCIS J., Sedimentary Rocks, New York, Harper and Row, Harper's Geoscience Series, 1957.

4) LEE, DAVID R. et G. THOMAS SALLEE, «A Method of Measuring Shape», Geographical Review, 1970, vol. 60 , no 4, p. 555.

5) BUNGe, WILliam, Theoritical Geography, Lund, Gleerup, 1962; en particulier le chapitre 111 «A Measure of Shape», p. 72-88.

6) BUNGE, WILLIAM, op. cit., p. 77.

7) BOYCE, RONALD R. et W. A. V. CLARK, «The Concept of Shape in Geography», Geographical Review, 1964, vol. 54, p. 561-572.

8) LEE, DAVID R. et G. THOMAS SALLEE, article cité, p. 555-556.

9) ibid., article cité, p. 555-563.

10) Recherche effectuée dans le cadre de la thèse de doctorat d'Etat d'ANDRÉ-LOUIS SANGUIN, intitulée: «La Suisse, essai de géographie politique» et inscrite à l'Université de Paris-Sorbonne (Paris 1V). 